\title{
ON FUNCTION THEORY ON SPACES WITH A LOWER RICCI CURVATURE BOUND
}

\author{
Tobias H. Colding and William P. Minicozzi II
}

\begin{abstract}
In this announcement, we describe some results of an ongoing investigation of function theory on spaces with a lower Ricci curvature bound. In particular, we announce results on harmonic functions of polynomial growth on open manifolds with nonnegative Ricci curvature and Euclidean volume growth.
\end{abstract}

\section{Introduction}

Twenty years ago Yau, [Y1], generalized the classical Liouville theorem of complex analysis to open manifolds with nonnegative Ricci curvature. Specifically, he proved that a positive harmonic function on such a manifold must be constant. This theorem of Yau was considerably generalized by Cheng-Yau (see $[\mathrm{ChY}]$ ) by means of a gradient estimate which implies the Harnack inequality. As a consequence of this gradient estimate (see [Ch]), one has that on such a manifold even a harmonic function of sublinear growth must be constant. In order to study further the analytic properties of these manifolds one would like to restrict the class of functions to be considered as much as possible while minimizing loss of information (cf. [CM2]). From the results of Cheng and Yau, it follows that a natural candidate is the class of harmonic functions of polynomial growth (note that they must be of at least linear growth). In fact, in his study of these functions, Yau was motivated to make the following conjecture (see [Y3], [Y4], and [Y5]; cf. [L1]):

Conjecture 0.1. (Yau). For an open manifold with nonnegative Ricci curvature the space of harmonic functions with polynomial growth of a fixed rate is finite dimensional.

We recall the definition of polynomial growth.

Definition 0.2. For an open (complete noncompact) manifold, $M^{n}$, given a point $p \in M$ let $r$ be the distance from $p$. Define $\mathcal{H}_{d}(M)$ to be the linear space of harmonic functions with order of growth at most $d$. This

Received August 17, 1995.

The first author was partially supported by NSF Grant DMS 9504994 and the second author by an NSF Postdoctoral Fellowship. 
means that $u \in \mathcal{H}_{d}$ if $u$ is harmonic and there exists some $C>0$ so that $|u| \leq C\left(1+r^{d}\right)$.

Our main result is the following.

Theorem 0.3. ([CM1]). Conjecture 0.1 is true if $M^{n}$ has Euclidean volume growth.

Remark 0.4. For each fixed rate of growth, we give explicit bounds on the dimension of the space of harmonic functions.

In fact, we give a description of the asymptotic behavior of polynomial growth harmonic functions in this case. We also give examples illustrating the subtleties that arise.

From the investigation initiated by the first author in [C1], [C2], and [C3], and later on further developed by the first author jointly with Cheeger in [CC1], [CC2], and [CC3], and finally the joint work of the first author with Cheeger and Tian in [CCT], we have a good understanding of the geometry of spaces with Ricci curvature bounded from below.

In $[\mathrm{CCM}]$, jointly with Cheeger we show that for each nontrivial independent linear growth harmonic function the tangent cone at infinity splits off a line. Examples of Kasue-Washio show that $M$ itself may not split (see $[\mathrm{KW}]$ ). As a consequence of this result proven in $[\mathrm{CCM}]$ and results of the first author in [C3], we have that a manifold with nonnegative Ricci curvature admitting the maximal number of independent linear growth harmonic functions must be isometric to Euclidean space (cf. [LT], [L2], and $[\mathrm{W}])$. That is, we get that $\mathbf{R}^{n}$ is the unique $n$-dimensional manifold with nonnegative Ricci curvature with the maximal number of independent linear growth harmonic functions.

Earlier important work on Conjecture 0.1 has been done by P. Li, P. Li and L.F. Tam, A. Kasue, J. Wang, and H. Wu. F.H. Lin has studied asymptotically conical elliptic operators and formulated similar problems on function theory on minimal submanifolds.

In a future paper, we plan on describing applications of this work, and considering some related questions (see [CM2]).

\section{Background}

We first recall the notion of Gromov-Hausdorff distance which we will use to measure the rough geometry of manifolds.

Definition 1.1. (see [GLP]). We will say that the Gromov-Hausdorff distance between two metric spaces $X_{1}$ and $X_{2}$ is less than $\epsilon$ if there exist 
maps $\Phi_{1}: X_{1} \rightarrow X_{2}$ and $\Phi_{2}: X_{2} \rightarrow X_{1}$ such that for every $a_{i}, b_{i} \in X_{i}$,

$$
\left|d\left(\Phi_{i}\left(a_{i}\right), \Phi_{i}\left(b_{i}\right)\right)-d\left(a_{i}, b_{i}\right)\right|<\epsilon
$$

and for $i \neq j$

$$
\left|d\left(\Phi_{j}\left(\Phi_{i}\left(a_{i}\right)\right), \Phi_{j}\left(\Phi_{i}\left(b_{i}\right)\right)\right)-d\left(a_{i}, b_{i}\right)\right|<\epsilon .
$$

Similarly, if $X$ is an unbounded metric space and $p \in X$, we can define pointed Gromov-Hausdorff convergence with base point $p$ (see [GLP]); this is essentially Gromov-Hausdorff convergence on bounded subsets.

Let $M^{n}$ be an open Riemannian manifold with nonnegative Ricci curvature. By Gromov's compactness theorem, [GLP], any sequence, $r_{i} \rightarrow \infty$, has a subsequence, $r_{j} \rightarrow \infty$, such that the rescaled manifolds $\left(M, p, r_{j}^{-2} g\right)$ converge in the pointed Gromov-Hausdorff topology to a length space, $M_{\infty}$. Such an $M_{\infty}$ is called a tangent cone at infinity of $M$.

$M^{n}$ is said to have Euclidean volume growth if there exists $p \in M$ and a positive constant $C$ such that $\operatorname{Vol}\left(B_{r}(p)\right) \geq C r^{n}$ for all $r>0$. Note that Bishop's volume comparison theorem implies that $\operatorname{Vol}\left(B_{r}(p)\right) \leq \mathrm{V}_{0}^{n}(1) r^{n}$ for all $r>0$, where $\mathrm{V}_{\Lambda}^{n}(r)$ denotes the volume of the ball of radius $r$ in the $n$-dimensional space form of constant sectional curvature $\Lambda$.

Examples of Perelman ([P1]; see also [CC2] for further examples) show that $M_{\infty}$ is not unique in general even if $M$ has Euclidean volume growth and quadratic curvature decay (cf. [C3] and [CT]).

However, in [CC1] it is shown that if $M$ has Euclidean volume growth, then every such $M_{\infty}$ is a metric cone. For this reason, we will model large balls in such a manifold by large balls centered at the vertex of a cone.

We also note that examples of Perelman (see [P2]) most likely can be modified to give examples of manifolds with nonnegative Ricci curvature, Euclidean volume growth and infinite topological type.

\section{Harmonic functions with polynomial growth}

In this section, we describe our results settling Conjecture 0.1 when $M$ has Euclidean volume growth. As a step towards this, we give a description of the asymptotic structure of polynomial growth harmonic functions in this case.

It is a classical result that the space of harmonic functions of polynomial growth on Euclidean space is spanned by the spherical harmonics. Recall that the spherical harmonics are the homogeneous polynomials whose restriction to every sphere centered at the origin is an eigenfunction of the spherical Laplacian. More generally, the harmonic functions of polynomial growth on a metric cone can be written as a linear combination of harmonic 
functions which separate variables (into the radial and cross-sectional directions). Further, they are homogeneous in the radial direction; it follows that the restriction to the cross-section gives an eigenfunction (where the eigenvalue depends on the dimension and the order of growth). We show that asymptotically this picture still holds in the general case of nonnegative Ricci curvature and Euclidean volume growth. That is, on many sufficiently large annuli, harmonic functions of polynomial growth will almost separate variables and be approximately homogeneous in the radial direction.

An important tool in the proof of Theorem 0.3 is to study for a harmonic function $u$ the rate of growth $U(r)$ of the average of $u^{2}$ on the sphere of radius $r$. See [CM1] for a precise definition.

We also describe some of the subtleties that arise in the case of Euclidean volume growth compared to the model case of a cone. In particular, in contrast to the model case, $U$ is not always monotone (see [CM1] for examples).

We note that if $M$ does not have Euclidean volume growth, there might not be any nontrivial polynomial growth harmonic functions.

Example 2.1. There exist manifolds with nonnegative Ricci curvature (in fact, positive sectional curvature) which admit no nontrivial polynomial growth harmonic function. In fact, one may round off a metric of the form $d r^{2}+r^{2 \alpha} d \theta^{2}$, where $\alpha<1$ and $d \theta^{2}$ is the standard metric on $\mathbf{S}^{n-1}$. This example was observed by Kasue in [K1].

Example 2.2. (Klembeck, $[\mathrm{Kl}]$, and cf. [Mo]). In the holomorphic case, there exists a Kähler metric on $\mathbf{C}^{n}$ of positive sectional curvature and quadratic curvature decay which does not admit any nonconstant holomorphic functions with polynomial growth.

\section{References}

[AL] M. Avellenada and F. H. Lin, Un théoreme de Liouville pour des équations elliptiques a coefficients périodiques, C.R. Acad. Sci. Paris t. 309, Serie 1 (1989), 245-250.

[CC1] J. Cheeger and T. H. Colding, Lower bounds on the Ricci curvature and the almost rigidity of warped products, Ann. of Math. (to appear).

[CC2] _ On the structure of spaces with Ricci curvature bounded below; I (preprint).

[CC3] - On the structure of spaces with Ricci curvature bounded below; II (to appear).

[CC4] _ Almost rigidity of warped products and the structure of spaces with Ricci curvature bounded below, C.R. Acad. Sci. Paris t. 320, Serie 1 (1995), 353-357.

[CCM] J. Cheeger, T. H. Colding, and W. P. Minicozzi II, Linear growth harmonic functions on complete manifolds with nonnegative Ricci curvature, J. Geom. and Funct. Anal. (GAFA) 5, no. 6 (1995), 948-954. 
[CCT] J. Cheeger, T. H. Colding, and G. Tian, Constraints on singularities under Ricci curvature bounds, (preprint).

[CT] J. Cheeger and G. Tian, On the cone structure at infinity of Ricci flat manifolds with Euclidean volume growth and quadratic curvature decay, Invent. Math. 118 (1994), 493-571.

[Ch] S. Y. Cheng, Liouville theorem for harmonic maps, Proc. Symp. Pure Math. 36 (1980), 147-151.

[ChY] S. Y. Cheng and S.-T. Yau, Differential equations on Riemannian manifolds and their geometric applications, Comm. Pure Appl. Math. 28 (1975), 333-354.

[C1] T. H. Colding, Shape of manifolds with positive Ricci curvature, Invent. Math. 124, Fasc. 1-3 (1996), 175-191.

[C2] _ Large Manifolds with Positive Ricci Curvature, Invent. Math. 124, Fasc. 1-3 (1996), 193-214.

[C3] _ Ricci Curvature and Volume Convergence, Ann. of Math. (to appear).

[C4] _ Stability and Ricci curvature, C.R. Acad. Sci. Paris t. 320, Serie 1 (1995), 1343-1347.

[CM1] T. H. Colding and W. P. Minicozzi II, Harmonic functions with polynomial growth, (to appear).

[CM2] , (in preparation).

[GW] R. Greene and H. Wu, Function theory on manifolds which possess a pole, Springer Lec. Notes in Math. 699 (1979).

[GLP] M. Gromov, J. Lafontaine and P. Pansu, Structures métriques pour les variétiés riemanniennes, Cedic/Fernand Nathan, Paris (1981).

[K1] A. Kasue, Harmonic functions of polynomial growth on complete manifolds, Proc. Symp. Pure Math. 54, Part 1, Ed. R. Greene and S.-T. Yau.

[K2] _ Harmonic functions of polynomial growth on complete manifolds II, J. Math. Soc. Japan, (to appear).

[KW] A. Kasue and T. Washio, Growth of equivariant harmonic maps and harmonic morphism, Osaka J. Math. 27 (1990), 899-928.

[Kl] P. Klembeck, A complete Kähler metric of positive sectional curvature on $\mathbf{C}^{n}$, Proc. Amer. Math. Soc. 64 (1977), 313-316.

[L1] P. Li, The theory of harmonic functions and its relation to geometry, Proc. Symp. Pure Math. 54, Part 1, Ed. R. Greene and S.-T. Yau.

[L2] _ Linear growth harmonic functions on Kähler manifolds with nonnegative Ricci curvature, Math. Res. Lett. 2 (1995), 79-94.

[LT] P. Li and L-F. Tam, Linear growth harmonic functions on a complete manifold, J. Diff. Geom. 29 (1989), 421-425.

[Mo] N. Mok, A survey on complete noncompact Kähler manifolds of positive curvature, Proc. Symp. Pure Math. 41 (1984), 151-162.

[P1] G. Perelman, (personal communication).

[P2] _ Construction of manifolds of positive Ricci curvature with big volume and large Betti numbers, (preprint).

[W] J. Wang, Linear growth harmonic functions on complete manifolds, Comm. Anal. Geom. 3, no. 4 (1995), 683-698.

[Wu] H. Wu, Polynomial functions on complete Kähler manifolds, Proc. Symp. Pure Math. 52, Part 2 (1991), 601-610. 
[Y1] S. T. Yau, Harmonic functions on complete Riemannian manifolds, Comm. Pure and Appl. Math. 28 (1975), 201-228.

[Y2] _ Some function-theoretic properties of complete Riemannian manifold and their applications to geometry, Indiana Univ. Math. Journal 25 (1976), 659-670.

[Y3] _ Nonlinear analysis in geometry, L'Enseignement Mathematique 2, no. 33 (1987), 109-158.

[Y4] _ _ Open problems in geometry, Proc. Symp. Pure Math. 54, Part 1, Ed. R. Greene and S.-T. Yau.

[Y5] __ Open problems in geometry, Chern - A great geometer of the twentieth century, Ed. S.-T. Yau, International Press, 1993.

Courant Institute of Mathematical Sciences, 251 Mercer Street, NEW YoRK, NY 10012

E-mail address: colding@cims.nyu.edu

Johns Hopkins University, 3400 N. Charles Street, Baltimore, MD 21218

E-mail address: minicozz@math.jhu.edu 